\title{
DISEÑO DE UNA APLICACIÓN ADAPTATIVA PARA MONITOREO REMOTO A TRAVÉS DE TECNOLOGÍAS MÓVILES
}

Andrés S. Barragán.

Ingeniero Electrónico, Especialista en Teleinformática. En la actualidad se encuentra vinculado a TowerTech Américas como Ingeniero en desarrollo de software. Bogotá, Colombia.

asbarraganv@unal.edu.co

\section{Carlos H. Ruiz}

Ingeniero Electrónico, Especialista en Teleinformática. Actualmente se desempeña como Profesional Especializado en el área de ingeniería en la Comisión de Regulación de Comunicaciones. Bogotá, Colombia.

ruizguzman@gmail.com

\section{Ernesto Gómez Vargas}

Ingeniero Electrónico Universidad Distrital "Francisco José de Caldas", Especialista en Telecomunicaciones Móviles Universidad Distrital Francisco José de Caldas, MSc. en Teleinformática Universidad Distrital Francisco José de Caldas, Estudiante del Programa de Doctorado de Ingeniería de la pontificia Universidad Javeriana, Docente de planta de la Universidad Distrital Francisco José de Caldas, Bogotá, Colombia. egomez@udistrital.edu.co

Tipo: Artículo de reflexión

Fecha de Recepción: Abril 5 de 2010 Fecha de Aceptación: Mayo 14 de 2010

\section{ADAPTIVE APPLICATION DESIGN FOR REMOTE MONITO- RING THROUGH MOBILE TECHNOLOGIES}

\begin{abstract}
Design considerations are presented regarding a mobile application that allows monitoring different processes at both residential and/or industrial levels using a simple, userfriendly interface. While these design considerations can be generally applied to monitoring residential and/ or industrial processes, this work focuses on electrical power consumption, whether residential or corporate. The use of the application by different users is presented according to different site configurations and enabled profiles. Likewise, the application takes into consideration Administrator access to parameter configuration from a Web interface. These elements are included in the design, taking into account software development methodologies that have been widely accepted in the industry.
\end{abstract}

Keywords: Data base, core, user interface, monitoring, RUP, mobile technologies.

\section{Resumen}

Se presentan las consideraciones asociadas al diseño de una aplicación móvil que permita mediante una interfaz sencilla y amigable con el usuario, la supervisión de diferentes procesos a nivel residencial y/o de industria. Si bien las consideraciones de diseño expuestas pueden ser aplicadas de manera general al monitoreo de procesos residenciales y/o industriales, la temática expuesta se encuentra enfocada al consumo de energía eléctrica, bien sea a nivel residencial o corporativo. Se contempla el uso de la aplicación por parte de diferentes usuarios, según diferentes configuraciones de sitios y perfiles que se encuentren habilitadas. Así mismo la aplicación toma en consideración el acceso a la configuración de parámetros por parte del Administrador, desde una interfaz Web. Tales elementos son incluidos en el diseño tomando en consideración metodologías de desarrollo de software de amplia aceptación en la industria.

Palabras clave: Base de datos, core, interfaz de usuario, monitoreo, RUP, tecnologías móviles. 


\section{INTRODUCCIÓN}

En la actualidad la supervisión y control de algunos procesos aún se realiza mediante computadores de escritorios o portátiles, a pesar de que los celulares más modernos, o teléfonos inteligentes, ofrecen la posibilidad de implementar cualquier tipo de comunicación, ya que el hardware no es una limitante como ocurría antes.

El control y supervisión mediante computadores de escritorio y/o laptops puede suponer ineficiencias, toda vez que, cargar con ellos puede llegar a ser incomodo en ocasiones, lo anterior a pesar de que actualmente se encuentran computadores portátiles pequeños. Además debe tenerse en cuenta que debe proveerse al equipo de una conexión a Internet para efectos de realizar el monitoreo, lo cual puede ser logrado a través de $\mathrm{WiFi}$, o por medio de un módem 3G para establecer la conexión por medio de las redes celulares, lo que supone mayores costos en cuanto a la contratación del servicio de acceso a Internet para estos dispositivos. El desarrollo en los terminales de telefonía móvil ha llevado a que muchas aplicaciones de monitoreo se estén orientado completamente a plataformas celulares de tal forma que se puedan aprovechar al máximo las características que ofrecen los teléfonos que se emplean para las comunicaciones a través de las redes móviles, además del hecho que muchos de ellos ya cuentan con planes para conectarse a Internet desde el terminal.

Si se mantiene la tendencia de la evolución de los celulares de alta gama, éstos poco a poco irán reemplazando cada vez más a los equipos portátiles en muchas tareas realizadas por el usuario promedio. Con esta tendencia las aplicaciones desarrolladas para ser utilizadas completamente a través de terminales móviles tendrán aún más demanda que las aplicaciones para equipos de escritorio.

\section{MARCO TEÓRICO}

El impulso que ha tenido la telefonía móvil, tanto a nivel mundial como en Colombia, hace posible el uso de dicho medio de comunicación para todos los procesos para los que antes era necesario un PC con una conexión a Internet a través de un cable. Esto permitirá que todo pueda ser supervisado en cualquier momento desde cualquier parte del mundo de una manera cómoda que no requerirá el traslado de grandes equipos, con lo cual podría brindarse diferentes tipos de soluciones desde i) evitar que se generen pérdidas en una empresa por falta de control sobre diferentes procesos hasta ii) brindar posibilidades a la población en general para que monitoree actividades convencionales en el hogar: el encendido de un bombillo, la apertura de una puerta, entre otros. La tecnología de los celulares y las redes celulares ha tenido su mayor crecimiento en los últimos años. Las redes celulares de tercera y cuarta generación permiten tener fácilmente acceso a Internet desde cualquier ubicación y el hardware con el que cuentan los dispositivos permite que se ejecuten aplicaciones robustas.

En relación con lo anterior, de acuerdo con las cifras oficiales publicadas por el Gobierno Nacional [1] [2], la evolución en la cantidad de usuarios del servicio de Acceso a Internet a través de redes móviles por suscripción (es decir, que pagan un cargo mensual para acceder al servicio), ha presentado crecimientos marcados en los últimos años, teniendo a Diciembre de 2010 un total de 1'708.633 suscriptores al servicio, lo cual representa un crecimiento de $85,3 \%$ respecto a diciembre de 2009 cuando la cifra fue de 921.830. Este comportamiento se ilustra en la Fig. 1. 


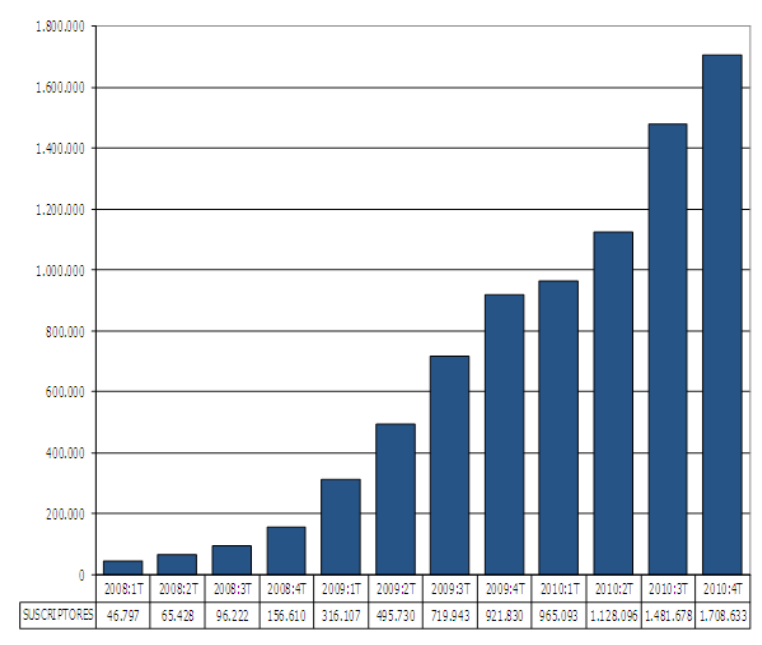

Fig. 1. Evolución de acceso a Internet a través de redes móviles por suscripción (asociado al pago de una mensualidad) [1], [2].

Ahora bien, para supervisión remota a través de redes móviles el enfoque se ha dado especialmente en el monitoreo de sistemas de telemedicina, por ejemplo como mecanismo de apoyo a personas con diabetes en el autocuidado de su condición para lo cual se han desarrollado sistemas en tiempo real basados en la transmisión y la retroalimentación de datos desde y hacia un teléfono móvil [3], o como plataforma inteligente de televigilancia de procesos de electrocardiografía sin pérdida de compresión del electrocardiograma (ECG) [4].

Por otra parte, también se han conocido experiencias aplicadas a la supervisión y control a nivel industrial mediante un teléfono móvil, utilizando un módem GSM conectado a un autómata programable o PLC, mediante el envío y la recepción de mensajes cortos de texto -SMS- (Short Message Service) [5].

Así mismo, actualmente se encuentran disponibles en el mercado diferentes ofertas de monitoreo y control remoto para la industria, orientadas a la conexión de los procesos a través de redes fijas [6]. No obstante, algunas empresas ofrecen soluciones que permiten realizar actividades desde una conexión a Internet móvil, como es el caso presentado en [7].

La Fig. 2 presenta un modelo general de sistema de supervisión de datos. En 1) se tiene el proceso a monitorear, 2) corresponde a la interfaz de comunicación entre el proceso que se monitorea y la red de datos (en este caso Internet), 3) corresponde a la red de Internet propiamente dicha, 4) se refiere a las bases de datos donde se almacena la información de los procesos monitoreados, a las cuales acceden los usuarios según se presenta en 5).

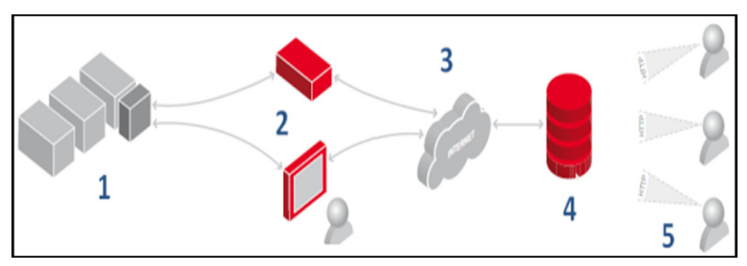

Fig. 2. Modelo de configuración de un sistema de monitoreo remoto [7].

Se plantea entonces que la aplicación para supervisión remota de varios procesos debe contar con la facilidad de uso y sencillez que ayude al usuario a monitorear procesos sin depender de diferentes aplicaciones. En tal sentido, la tecnología actual de los celulares permite el desarrollo de aplicaciones que permiten la integración de varios protocolos sin que se vea afectada la velocidad y rendimiento de la aplicación.

Se estima que el desarrollo de esta aplicación tendría un impacto económico, ya que se puede integrar a los sistemas de supervisión actuales sin que exista la necesidad de realizar cambios sobre los mismos. Teniendo en cuenta que las infraestructuras para supervisión de procesos ya están probadas y son muy robustas, la integración de una aplicación de este tipo evitaría cambios innecesarios que pueden llegar a afectar el funcionamiento actual de los mismos.

\section{ANÁLISIS DE REQUERIMIENTOS}

Para efectos de acotar los requerimientos [8] de la aplicación, y de acuerdo con lo ex- 
puesto en la sección anterior, el proceso de diseño estará orientado al monitoreo del consumo de energía eléctrica, bien sea a nivel residencial o corporativo. De manera general, se prevé que los sitios a monitorear alimenten una base de datos con información de las mediciones. Se estima entonces que se puede contar con una base de información centralizada para cada municipio, que a su vez estaría conectada a servidores ubicados en diferentes puntos del municipio, los cuales tendrían la función de concentrar la información de los predios con los que tengan relación. La Fig. 3 presenta de manera gráfica estas consideraciones para la ciudad de Bogotá. En dicha figura, "1" representa la base de datos centralizada, "2" corresponde a las bases de datos ubicadas en diferentes localidades, y "3" identifica los predios o ubicaciones geográficas a monitorear. Nótese que, para efectos de obtener la consulta deseada, el terminal móvil establecería una comunicación, bien sea con la base de datos centralizada, o con alguna de las ubicadas en las localidades de un municipio dado, de acuerdo con la configuración realizada.

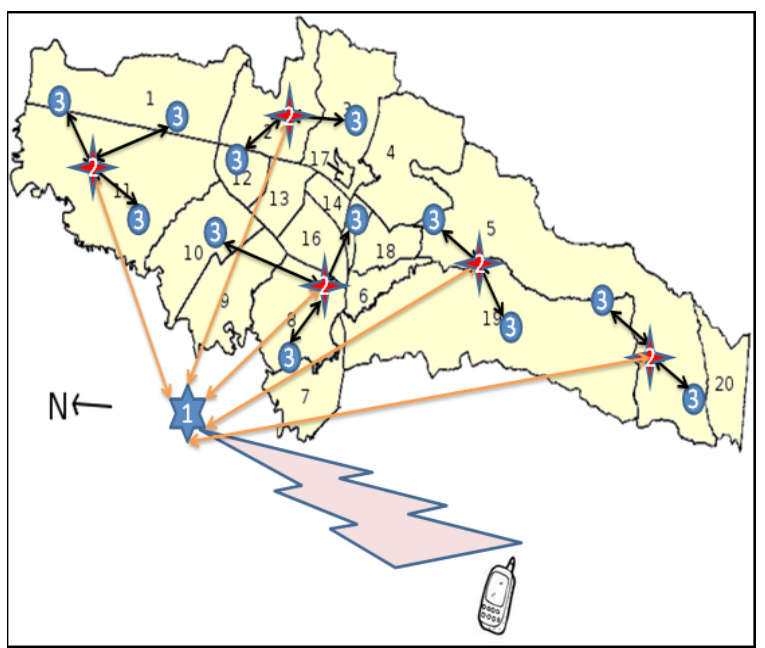

Fig. 3. Descripción de funcionamiento de la aplicación.

En relación con lo anterior, se tienen en cuenta las siguientes especificaciones para la aplicación:
- Debe ser posible para el usuario elegir el sitio a monitorear, de acuerdo con la habilitación que se haya hecho para el efecto.

- La aplicación debe permitir visualizar el valor actual de la medición. Se considera un intervalo de muestreo de diez (10) minutos.

- La aplicación debe permitir conocer el valor del consumo acumulado diario de la ubicación a monitorear.

- También debe ser posible configurar un comparativo del consumo en días, detallando la franja horaria que se desea comparar.

- El sistema debe tener disponible la opción de consultar el acumulado del consumo que se ha registrado en el mes.

- Deberá ser posible consultar información de hasta 60 días posteriores a la medición.

- La presentación de la información será realizada en gráfico o tabla, según la elección del usuario.

- La aplicación incluirá una opción de configuración de alertas de consumo, es decir, intervalos mínimo y máximo para alertas por comportamiento del contador de energía. Las mismas podrían ser configuradas según necesidades (por ejemplo de acuerdo al estrato).

Se estima necesario contar con una validación de ingreso al sistema. Así mismo debe realizarse una configuración de los procesos (casas, apartamentos, industrias, entre otros) a monitorear, a efectos de establecer el acceso a un servidor o servidores de manera predeterminada.

Finalmente, el sistema debe permitir conocer en todo momento el estado del proceso.

\section{MODELO FUNCIONAL}

El resultado de aplicar UML [9] [10] para modelar los requerimientos enunciados en el numeral anterior se presenta en la Fig. 4. 


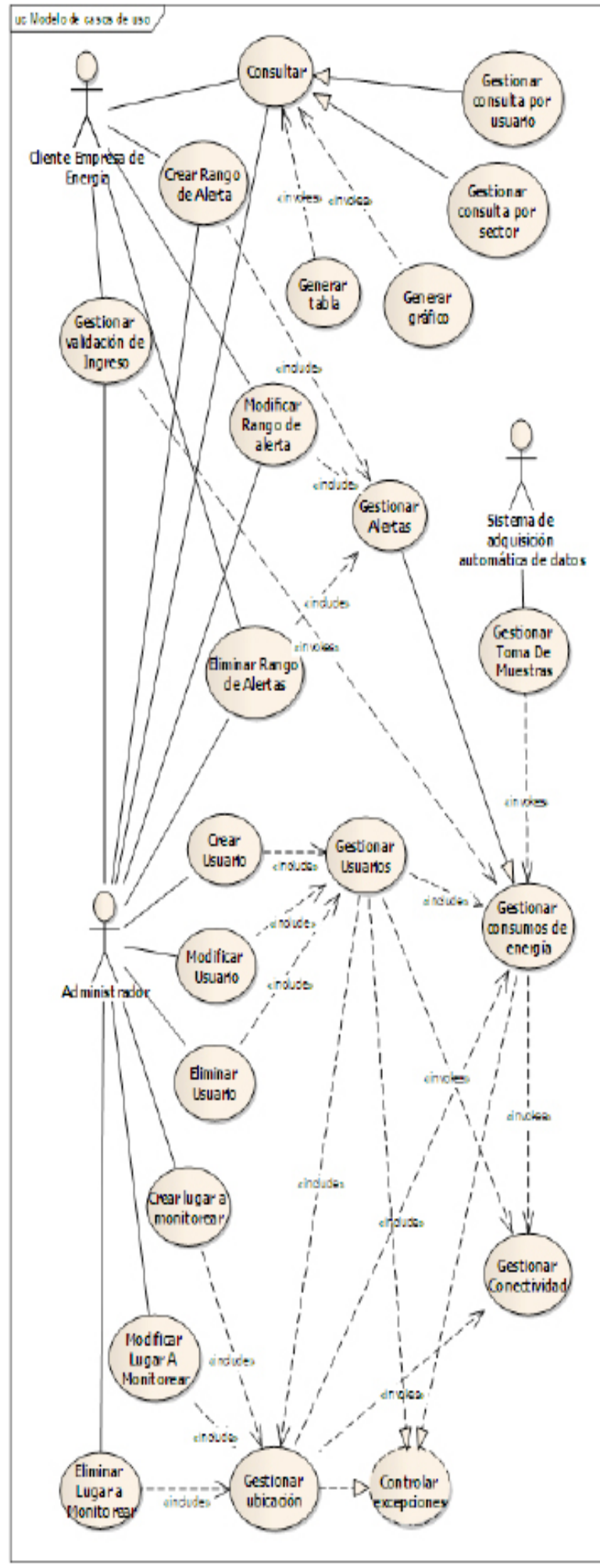

Fig. 4. Diagrama de casos de uso para la aplicación diseñada.

\section{MODELO ESTRUCTURAL BÁSICO}

Clases diseñadas para la aplicación móvil. A partir de los casos de Uso se llegaron a las siguientes clases para el servidor.La primera clase es "User" (Fig. 5), define los dos tipos de usuarios que existirán en el sistema, Administrador y Consumidor.

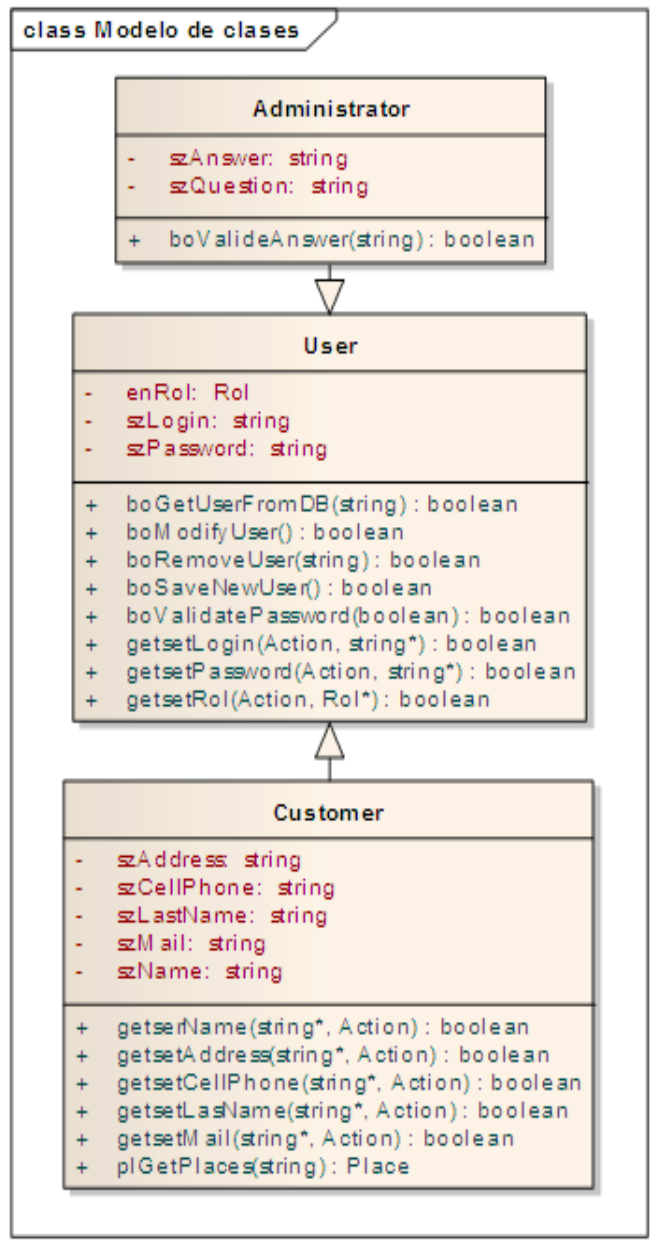

Fig. 5. Clases User, Customer y Administrator.

En las clases mostradas en la Fig. 5, es importante destacar los métodos boGetUserFromDB, boModifyUser, boRemoveUser y boSaveNewUser, que se encargan de armar las cadenas de conexión a la base de datos necesarias para obtener, modificar, eliminar y guardar usuarios respectivamente.

La siguiente es la clase "Place" (Fig. 6), está 
destinada a administrar la información de los diferentes sitios que se van a monitorear.

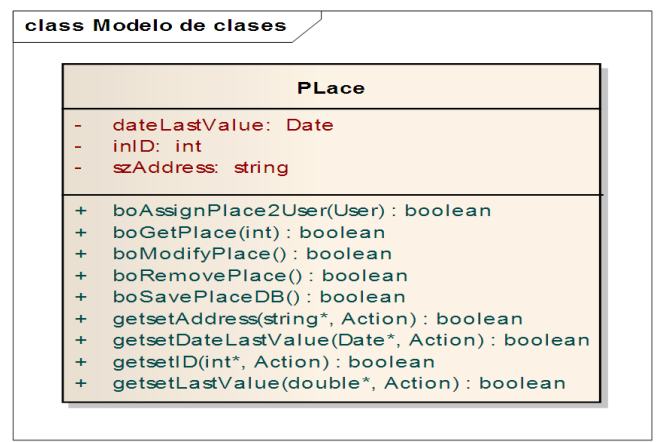

Fig. 6. Clase Place.

Dentro de ella es importante destacar el método "boAssignPlace2User", que se encarga de asociar un usuario a un lugar, de tal forma que el usuario pueda consultar los datos del lugar configurado.La estructura "GetSamples" (Fig. 7) esta destinadaa importar los datos capturados por la interfaz del hardware esto se puede realizar a través del puerto USB o de una conexión LAN (en caso de que las muestras se estén tomando en un lugar retirado).

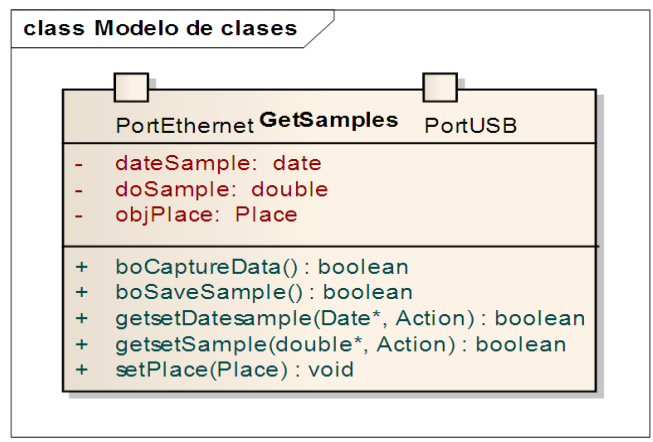

Fig. 7. Clase GetSamples. Elaboración propia.

Aquí, "boCaptureData” es el encargado de conectarse con la interfaz y obtener el valor de la muestra. Esta clase utiliza el objPlace, para determinar donde se debe conectar para obtener las muestras.

La clase "DataBase" (Fig. 8) administra y gestiona las conexiones a la base de datos.

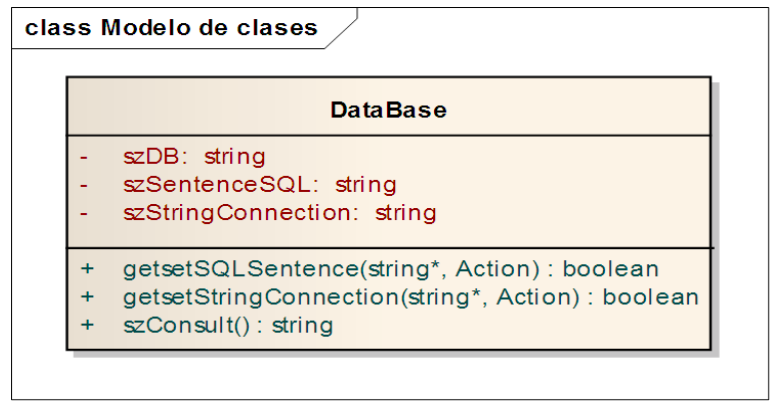

Fig. 8. Clase DataBase.

En este caso quien realiza la consulta es "szConsult". Además de realizar la conexión y obtener la respuesta de la consulta, está encargado de almacenar utilizando Logs, todos los errores y excepciones que se presenten en la conexión, de tal forma que estos no sean visualizados por el usuario final y puedan ser consultados posteriormente por el administrador. "ExportData" (Fig. 9) es una interfaz que se debe implementar dependiendo de la forma en que se vaya a obtener la información (web service, Servlet, TCP, etc.) remotamente.

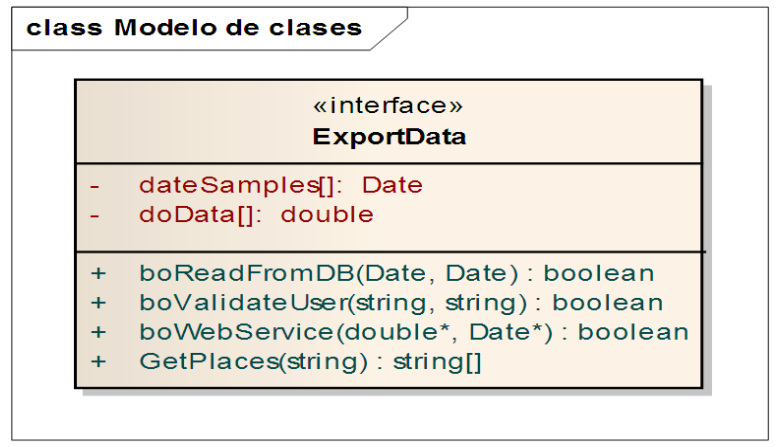

Fig. 9. Interfaz ExportData.

"BoValidateUser", valida el usuario que se intenta conectar desde el dispositivo móvil. El método boWebService trabaja lo relacionado con la adquisición de los datos desde la base de datos y el envío al cliente móvil, y GetPlace entrega los elementos necesarios para la adquisición de todos los lugares asociados a un usuario. 
Clases diseñadas para la aplicación del servidor. A partir de los casos de Uso se establecieron las siguientes clases para la aplicación Web.

"LocalDBSamples" (Fig. 10) está encargada de guardar en un archivo con formato $\mathrm{XML}$, las muestras obtenidas desde el servidor. Se guardará un número específico de muestras, y a medida que se agreguen más muestras, se eliminarán los registros más antiguos.

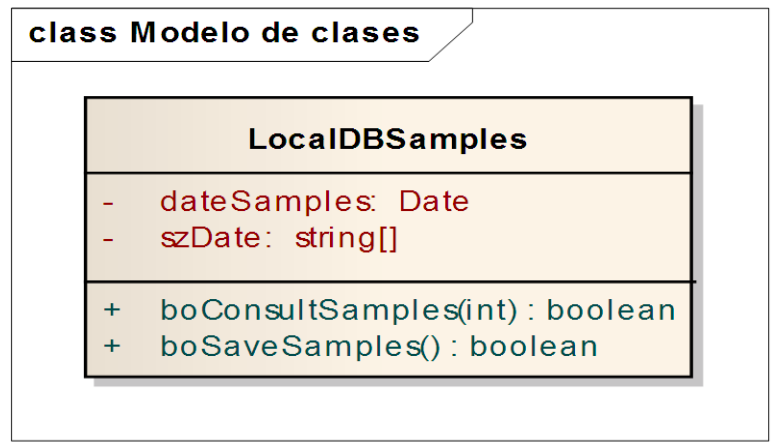

Fig. 10. Clase LocalDBSamples.

Mediante "boConsultSamples", se permite consultar los últimos registros almacenados en el archivo.

"LocalDBPlaces" (Fig. 11) es la encargada de almacenar en el celular todos los lugares asociados al usuario. Estos son guardados en un archivo con formato XML.

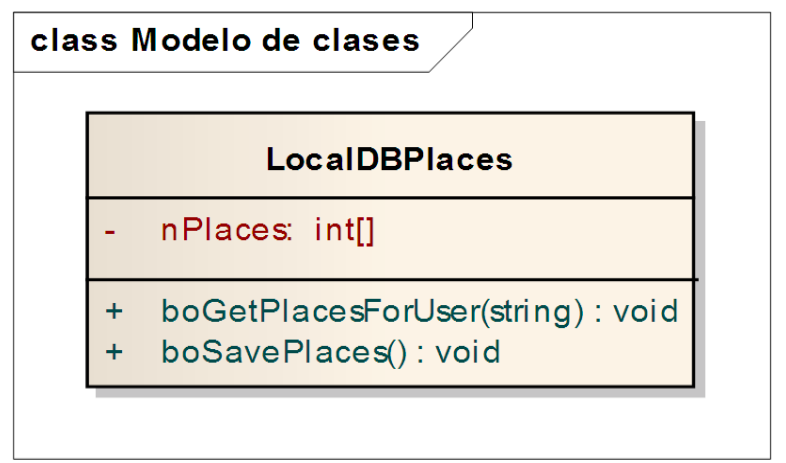

Fig. 11. Clase LocalDBPlaces.

La clase "DrawHistorical" (Fig. 12) muestra en pantalla un gráfico de todas las capturas tomadas en un rango de tiempo.

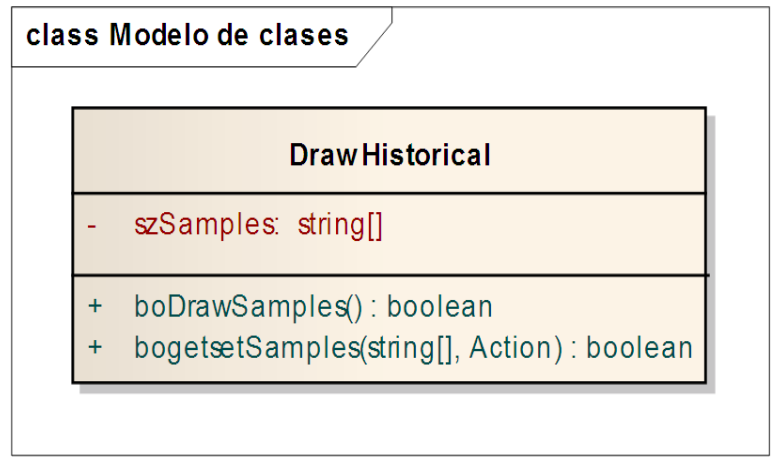

Fig. 12. Clase DrawHistorical.

La clase "ShowData" (Fig. 13) visualiza en una tabla toda información de las muestras tomadas incluyendo fecha y valores.

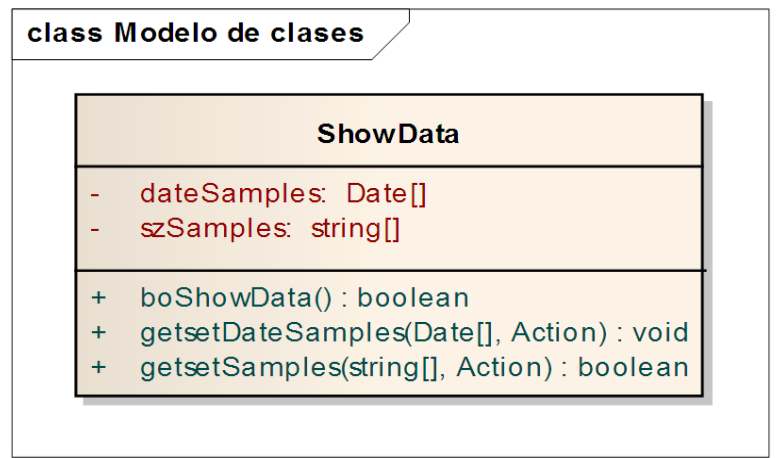

Fig. 13. Clase ShowData.

"ImportData" (Fig. 14) es un interfaz que, dependiendo de cómo se implemente, puede comunicarse mediante diferentes técnicas (TCP, HTTP, web services, Servlets).

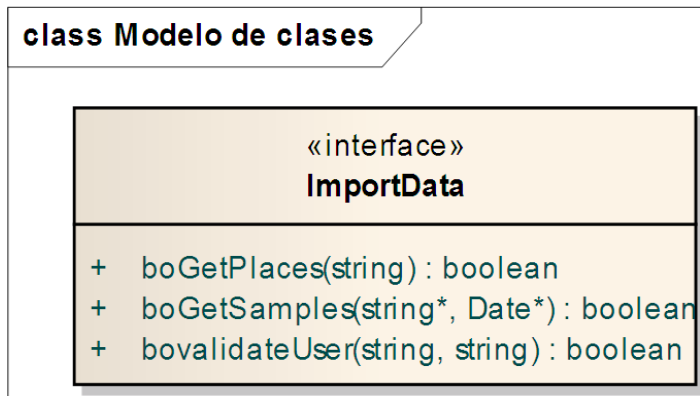

Fig. 14. Interfaz ImportData. 
"Alerts" (Fig. 15) administra las diferentes alertas que se configuren en la terminal.

\begin{tabular}{|c|c|}
\hline \multirow{2}{*}{\multicolumn{2}{|c|}{ class Modelo de clases }} \\
\hline & \\
\hline- & $\begin{array}{l}\text { dolevelD: double } \\
\text { nIDD: int }\end{array}$ \\
\hline $\begin{array}{l}+ \\
+ \\
+\end{array}$ & $\begin{array}{l}\text { boGetAlerts(): boolean } \\
\text { boSaveAlert(double, int) : boolean } \\
\text { nEvaluateAlert(double) : int }\end{array}$ \\
\hline
\end{tabular}

Fig. 15. Clase Alerts.

\section{BASES DE DATOS PARA LA APLICA- CIÓN}

Como se expuso en las secciones anteriores del presente artículo, para el desarrollo de la aplicación se requiere diseñar una base de datos que almacene la información de configuración de sitios, usuarios y mediciones del sistema, de acuerdo con características particulares en cada caso.

Para el efecto, se empleó el Modelo Entidad - Relación [11], a partir del cual se definió la necesidad de contar con seis (6) tablas, de acuerdo con lo mostrado en la Fig. 16.

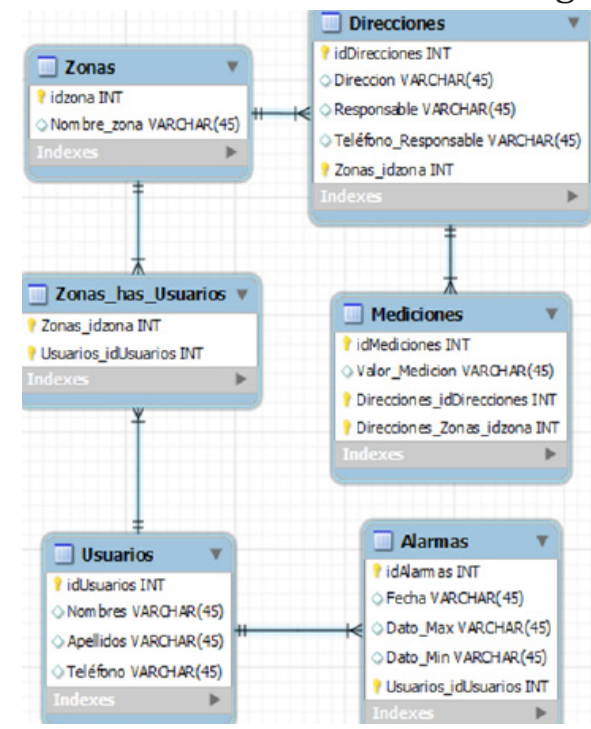

Fig. 16. Modelo para la capa de persistencia.
Interfaz de la aplicación móvil. A continuación se describen cada una de las pantallas que se usarán en el flujo de la aplicación. La primera pantalla que se va a encontrar al iniciar la aplicación (Fig. 17) es la de "login", en esta pantalla se solicitara el nombre de usuario y contraseña, se enviara la transacción al servidor para la validación del usuario y se permitirá o no el ingreso a las opciones.

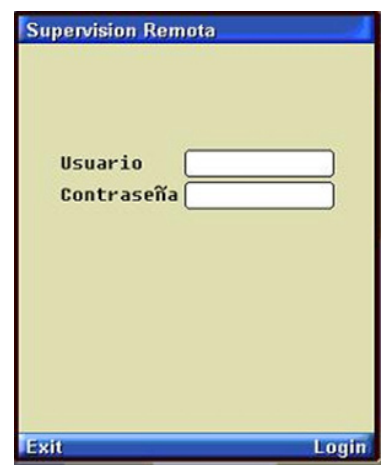

Fig. 17. Pantalla de login de la aplicación.

Seguidamente se enviará al servidor una transacción solicitando los lugares que tiene asociado el usuario. Estos lugares serán mostrados en un menú (Fig. 18).

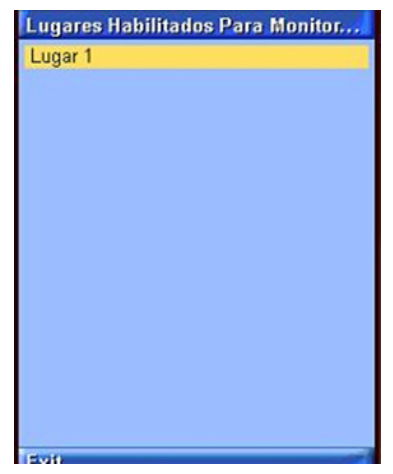

Fig. 18. Menú en donde se selecciona el lugar que se desea monitorear.

Luego de seleccionar un lugar se muestra un menú con las diferentes opciones que se pueden trabajar sobre el lugar seleccionado (Fig. 19). 


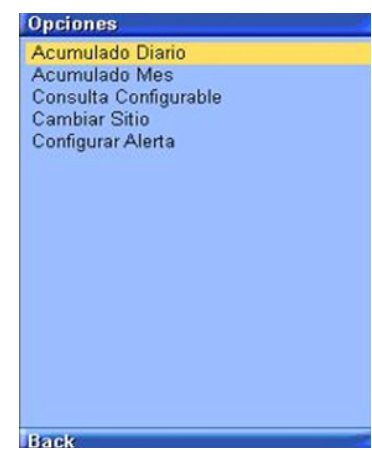

Fig. 19. Diferentes opciones para monitorear un lugar específico.

Si se selecciona la opción "Acumulado Diario" o "Acumulado Mes" se solicitará confirmar el modo en que se desea la visualización, como se muestra en la Fig. 20. Si se selecciona la opción cambiar sitio se retorna al menú donde se encuentra la lista de sitios que se tiene asociado el usuario para monitorear. Seleccionando la última opción "Configurar Alerta", se genera el formulario de la Fig. 21.

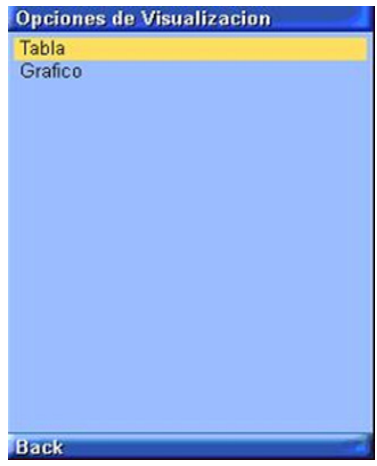

Fig. 20. Diferentes opciones de visualización de los datos.

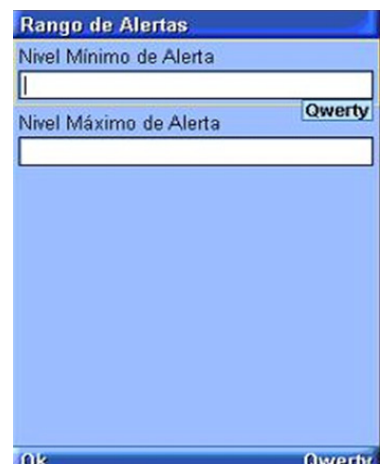

Fig. 21. Formulario para configuración de las alertas. Los dos tipos posibles de visualización apa- recen en la Fig. 22 y Fig. 23.

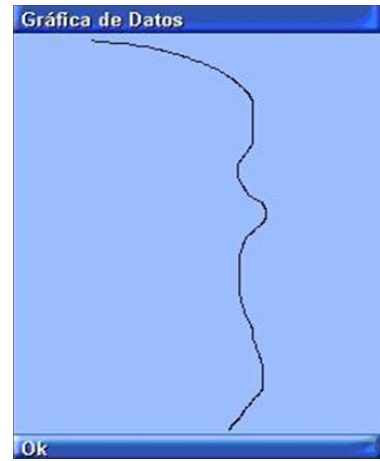

Fig. 22. Grafica de datos.

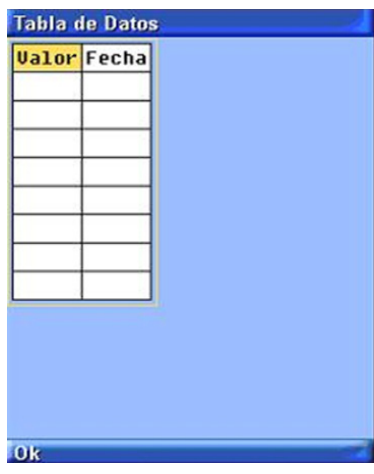

Fig. 23. Clase database.

Interfaz de la aplicación de administración. De igual modo que en la sección anterior, se presenta el prototipo funcional para la aplicación ubicada en el servidor, esta ayudará al administrador del Sistema en la configuración del mismo. En primer lugar debe validarse el ingreso (Fig. 24).

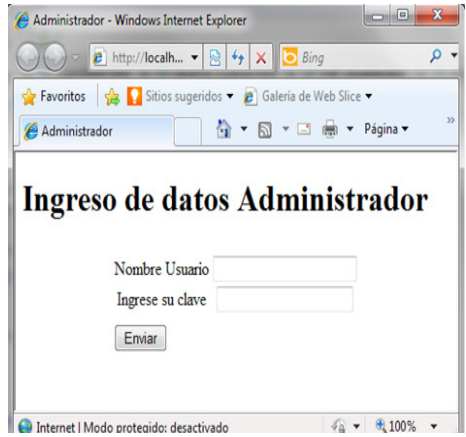

Fig. 24. Ingreso del Administrador al Sistema.

Luego de ingresar al sistema de manera exitosa, se presentará una pantalla con las 
acciones disponibles para el administrador, según se ilustra en la Fig. 25.

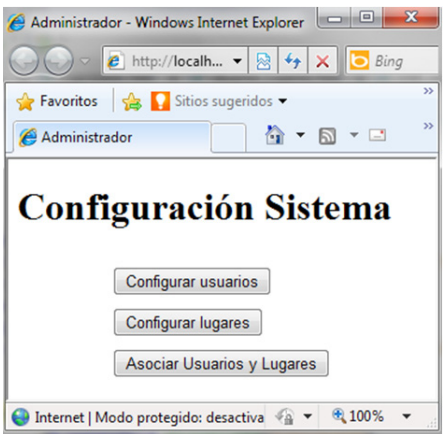

Fig. 25. Menú de selección de actividades.

Dentro de las posibilidades del administrador se tienen las de Crear, Modificar y Eliminar usuarios y lugares. Las pantallas de ingreso a estos aspectos se presentan en las Fig. 26 y 27 , respectivamente.

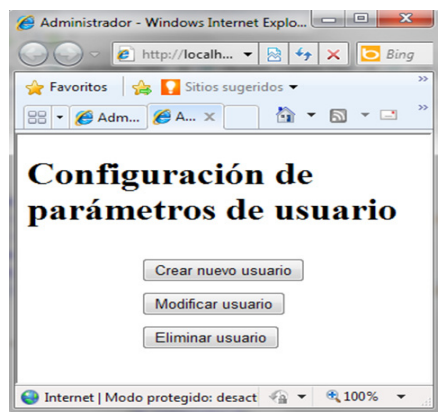

Fig. 26. Configuración de parámetros de usuario.

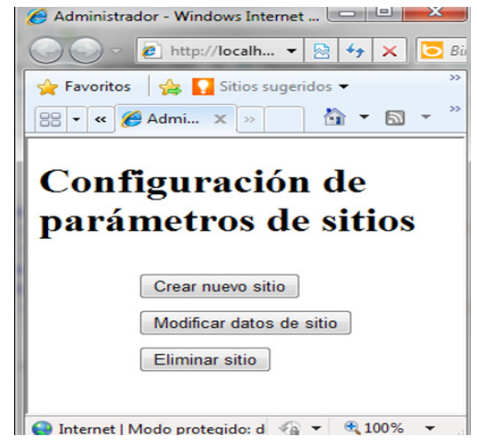

Fig. 27. Configuración de parámetros de sitios.

\section{CONCLUSIONES}

El desarrollo de la temática expuesta en el presente artículo, permite extraer las si- guientes conclusiones:

A partir de la evolución identificada en el presente estudio respecto de la evolución en las conexiones de acceso a Internet a través de redes móviles, y considerando que cada vez más las personas desean contar con información fiable y de fácil acceso respecto de diferentes temas, es claro que el diseño realizado aportará elementos importantes a la satisfacción de las necesidades tanto a nivel de industria como a nivel residencial en lo que tiene que ver con el monitoreo remoto de procesos.

Haber llevado a cabo una definición precisa de los requerimientos de la aplicación, desde el inicio del proceso de diseño, claramente ayudó a limitar el alcance del mismo y a definir las limitantes que podría tener la aplicación en cuanto a funcionalidad.

En relación con lo anterior, debe destacarse la importancia de incluir dentro de los requerimientos, la realización de procesos de validación de usuarios al sistema, tanto a nivel de administradores vía Web, como para los usuarios consumidores de la aplicación desde los terminales móviles, lo cual apunta a garantizar la integridad de la información y del sistema en sí mismo.

Cabe anotar que el proceso de realización del modelado estructural se facilitó al tomar como referencia el diagrama de casos de uso; de allí, se evidenció la necesidad de contar con clases específicas para la aplicación Web y para la aplicación móvil.

Para futuros diseños se deja la posibilidad de cifrar la comunicación entre el celular y el servidor, a efectos de evitar que la información pueda ser interceptada o manipulada por terceros.

Finalmente se concluye que se alcanzó el objetivo de diseñar una aplicación móvil que permita, mediante una interfaz sencilla y amigable con el usuario, la supervisión de diferentes procesos residenciales y/o de aplicación en la industria. 


\section{Referencias Bibliográficas}

[1] Información de los años 2008 y 2009 obtenida del Sistema de Información Unificado del Sector de las telecomunicaciones (SIUST), [en línea]. Consultado en Marzo 10 de 2010, disponible en:www.siust.gov.co.

[2] Cifras para el año 2010 extraídas de la página Web del Ministerio de Tecnologías de la Información y las Comunicaciones, [en línea]. Consultado en Febrero 15 de 2010, disponible en:www.mintic.gov.co/indus_cifras. asp

[3] A. Farmer; O. Gibson; P. Hayton; et al, "A real-time, mobile phone-based telemedicine system to support young adults with type 1 diabetes", British Computer Society, Boston, June 2-4, USA, 2009.

[4] F. Sufi; O. Fang; S. Mahmoud;I. Cosic, "A Mobile Phone Based Intelligent Telemonitoring Platform". International Summer School and Symposium on Medical Devices and Biosensors". MIT, Boston, USA, Sept.4-6, 2006.

[5] [5] C. Boter; Sánchez, R. Laia, "Sistema de Control y Supervisión remota basada en telefonía móvil
GSM". Departamento de Ingeniería Electrónica. Universidad Politécnica de Cataluña, 2009.

[6] A. García A;B. Curto; V. Moreno, "Software de Supervisión Remota para un Sistema de Control Distribuido". Departamento de Informática y Automática. Universidad de Salamanca, España, 2009.

[7] Adaptación de la figura presentada en el brochure de los productos com.tom, fabricados por la empresa Beck,[en línea]. Consultado en Marzo 20 de 2010, disponible en: www.beck-ipc. com/en/company/press/releases.asp.

[8] P. Kruchten, the rational unified process an introduction, editorial Addison Wesley, 2001.

[9] X. Grau, desarrollo orientado a objetos con UML, [en línea]. Consultado en Marzo 21 de 2010, disponible en:www.clikear.com/manuales/uml/ index.aspx

[10] R. Alarcón, diseño orientado a objetos con UML, editorial grupo EIDOS, España. 2000.

[11] C. Date, introducción a los sistemas de bases de datos, quinta edición, editorial Addison-Wesley, 1993. 